\title{
Vicente Huidobro oder ein avantgardistisches Schreiben in französischer und spanischer Sprache
}

Beschäftigen wir uns an dieser Stelle zunächst mit Vicente Huidobro, zweifellos einer der spannendsten Protagonisten innerhalb der lateinamerikanischen Avantgarden und beginnen mit einem kleinen biographischen Ausblick!

Der Lyriker und Intellektuelle wurde am 10. Januar 1893 in Santiago de Chile geboren und verstarb am 2. Januar 1948 im chilenischen Cartagena. Seine wichtigsten Biographeme lassen sich vielleicht so am besten zusammenfassen: Er entstammte einer alten chilenischen Großgrundbesitzerfamilie, besuchte ein exzellentes Jesuitenkolleg, heiratete 1911 standesgemäß, gründete 1912 die Literaturzeitschrift Musa Joven und brach 1916 nach Europa auf, genauer: nach Paris. Er lebte einige Jahre in der französischen, dann in der spanischen Hauptstadt und knüpfte wichtige literarische Freundschaften. Zusammen mit dem mit ihm befreundeten Guillaume Apollinaire und Pierre Reverdy gab er in Paris 1917 die Zeitschrift Nord-Sud heraus.

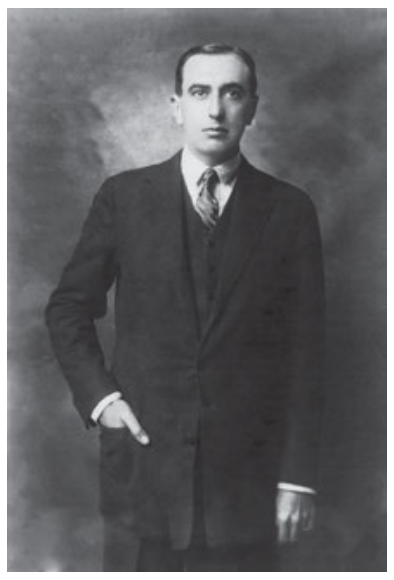

Abb. 56: Vicente Huidobro (Santiago de Chile, 1893 Cartagena, Region Valparaíso, 1948).

Sein wohl erster Besuch in Madrid im Jahr 1918 gilt als Auslöser der bald nach Argentinien verpflanzten avantgardistischen Bewegung des „Ultraísmo“. Er verfasste seine Gedichte nun in spanischer wie in französischer Sprache. Huidobro setzte sein Studium in Berlin und Fribourg fort, ging 1920 aber wieder nach Paris zurück, wo er Mitglied von Dada Paris und damit der europäischen Avantgarde wurde. 1925 trat er die Rückreise nach Chile an und stellte sich in seinem Heimatland als Präsidentschaftskandidat vor. Ein auf ihn verübtes Attentat und der 
Skandal wegen seiner Liebschaft mit einer minderjährigen Schülerin beendeten die politische Karriere und seinen Aufenthalt in Chile. 1928 entführte er seine Geliebte nach Paris. Den Spanischen Bürgerkrieg erlebte er auf Seiten der Republik als Pressekorrespondent. 1945 kehrte er endgültig nach Chile zurück, wo er wenige Jahre später verstarb.

Vicente Huidobro hat als Dichter ein vielbändiges lyrisches Werk, aber auch mehrere Romane und zahlreiche Prosaschriften verfasst. Er verstrickte sich in zum Teil beißende Polemiken, insbesondere mit seinem Landsmann, dem Lyriker Pablo Neruda, ${ }^{1}$ machte sich vor allem aber als Begründer des avantgardistischen Creacionismo einen Namen. Er selbst datierte die Entstehung seiner „Allgemeinen ästhetischen Theorie“ auf das Jahr 1912, was mit Blick auf die Entstehung avantgardistischer Bewegungen insbesondere in Lateinamerika als sehr früh eingeschätzt werden muss. Ohne jeden Zweifel war diese Theorie bereits vor seinem ersten Europaaufenthalt noch in Chile entstanden. Huidobro hatte sie bereits zum Teil ausformuliert und erstmals in frühen Gedichten seines Debütbandes Canciones en la noche 1913 erprobt:

Der Grundgedanke des Creacionismo, den er in zahlreichen Manifesten und Polemiken formuliert hat, ist die Gleichsetzung von Poesie und absoluter Schöpfung: „Der Dichter lässt die Dinge der Natur das Leben wechseln; mit seinem Netz holt er all das hervor, was sich im Chaos des Namenlosen bewegt, spannt elektrische Drähte zwischen den Worten und beleuchtet auf einmal unbekannte Winkel, so dass diese ganze Welt in unerwartete Phantasmen explodiert.“2

Huidobro verstand es, seine ästhetische Theorie pointiert auf den Punkt zu bringen und deren vier Grundprinzipien aufzulisten: Der Dichter müsse erstens die Dinge vermenschlichen, er müsse zweitens das Undeutliche präzisieren, er müsse drittens das Abstrakte konkret und das Konkrete abstrakt machen und schließlich viertens das, was an sich allzu poetisch ist, um vom Dichter geschaffen zu werden, durch Veränderung seines üblichen Wertes in etwas Geschaffenes verwandeln. Dies sind sehr allgemeine Prinzipien, gewiss, aber sie bilden doch die Leitlinien einer Schöpfung, die im Creacionismo ihren künstlerischen Ausdruck fand. ${ }^{3}$

1 Vgl. die Abrechnung Nerudas mit Huidobro in seinen Memoiren; Neruda, Pablo: Vicente Huidobro. In (ders.): Confieso que he vivido. Memorias. Barcelona - Caracas - México: Editorial Seix Barral 1974, S. 392-395.

2 Reichardt, Dieter: Lateinamerikanische Autoren. Literaturlexikon und Bibliographie der deutschen Übersetzungen. Tübingen: Erdmann 1972, S. 311.

3 Vgl. ausführlicher hierzu die Frankfurter Dissertation von Hopfe, Karin: Vicente Huidobro, der Creacionismo und das Problem der Mimesis. Tübingen: Narr 1996. 
Nach der Veröffentlichung seines ersten Gedichtbandes darf als ästhetisch frühe Einlösung von Huidobros Prinzipien wohl seine Dichtung Adán (1916) gelten, nach der Huidobro in seinen folgenden Werken noch weit mehr wagte und dazu seine Lyrik von nun an in spanischer, aber auch in französischer Sprache vorlegte, wie etwa Horizon carré von 1917 oder Tour Eiffel von 1918. Im selben Jahr erschienen auch die spanischsprachigen Gedichtbände Ecuatorial oder seine Poemas árticos. Huidobro experimentierte mit verschiedenen graphischen Elementen, ließ Zeilen verrutschen, sich verbiegen, kaskadenartig abfallen: Der chilenische Dichter beabsichtigte, nach dem Vorbild des Kubismus seine Dichtung mit den verschiedensten Künsten von Auge und Ohr in Verbindung zu setzen.

1931 erschien dann sein lyrisches Hauptwerk Altazor o el viaje en paracaídas („Altazor oder die Reise im Fallschirm“), dessen Entstehung bis in das Jahr 1919 zurückreicht. Ein lyrisches Werk in sieben Gesängen - wie Dieter Reichardt betonte, ein „faszinierendes Kompendium von Möglichkeiten, Grenzen und Widersprüchen der avantgardistischen Lyrik“.. Es ist eine Dichtung von außerordentlicher Sprachkraft, großem Erfindungsreichtum und radikalen Experimenten mit neuartigen, zum Teil von Huidobro selbst erdachten Wortschöpfungen und originellen Sprachspielen. Deutlich ist in diesem bis an die Grenzen des Verstehbaren gehenden Text das Erbe des Dadaismus, eine radikale Sprachgewalt, eingebettet in die Erfahrungen des französischen Surrealismus, den wir in unserer Vorlesung noch zu besprechen haben.

Auch die Prosaschriften Vicente Huidobros verdienten eine nähere Beschäftigung, ebenso sein Pamphlet Finis Brittania (1923) gegen den englischen Kolonialismus und seine Romane, die zum Teil mit graphischen Versatzstücken arbeiten und die Grenzen der Prosa in Arbeiten aufzeigen, die sich in Richtung der späteren Graphic Novel bewegen. Bereits 1934 schilderte er in seinem Roman La próxima die Schrecken und Zerstörungen eines totalen Krieges, so dass auch das für die historischen Avantgarden, für die Futuristen wie die Dadaisten, zentrale Thema des Krieges bei ihm nicht fehlte. Schon wenige Jahre später sollte der Krieg dann in einem barbarisch entfesselten Nazi-Deutschland ein totaler werden. Bis zu seinem Lebensende schrieb Huidobro unermüdlich und veröffentlichte noch in seinem letzten Lebensjahr 1948 seine Ultimos poemas.

Versuchen wir in der Folge, diesen Giganten der lateinamerikanischen Avantgarde in unserer Vorlesung zumindest exemplarisch darzustellen! Es sollen einige der für unsere Fragestellungen besonders repräsentativen Teile seines Gesamtwerks vorgestellt und nicht so sehr nach dessen innerer ästhetischer Entwicklung gefragt werden. Wir können daher auch nicht detailliert Huidobros Opus

4 Reichardt, Dieter: Lateinamerikanische Autoren, S. 312. 
magnum, seinen Dichtungsband Altazor von 1931, untersuchen, sondern wollen vor allem die Beziehungen zwischen hispanoamerikanischer und europäischer Avantgarde mit Blick auf die jeweils spezifischen Schreibformen beobachten.

Wir konnten in unserer kurzen Aufstellung der Lebensdaten bereits sehen, dass bestimmte Schaffensbereiche bei Vicente Huidobro schon vor dem direkten Kontakt mit den europäischen, speziell den französischen Avantgarden noch in seiner Zeit in Santiago de Chile entwickelt wurden. Bei alledem sollten wir nicht in die Falle tappen - wie dies meines Erachtens etwa Dieter Janik tat -, Huidobro in seiner ästhetischen Entfaltung ausschließlich vor dem Hintergrund der europäischen Avantgarden zu verstehen und zu beleuchten. Wie zuvor bei Alfonso Reyes wollen wir bei Vicente Huidobro die Eigenentwicklung spezifischer Avantgarden in Lateinamerika respektieren und nicht nach irgendwelchen ,Abweichungen' oder ,Besonderheiten' bezüglich der europäischen Avantgarden fragen. Denn diese sind keineswegs das Maß aller Dinge und schon gar keine Grundlage für eine einzige Theorie der Avantgarde, welche von Europa aus der Welt übergestülpt würde.

Harald Wentzlaff-Eggebert - der wohl als der beste deutschsprachige Kenner der Avantgarde-Literatur Hispanoamerikas in der zurückliegenden Epoche bezeichnet werden darf - hat mit guten Gründen darauf aufmerksam gemacht, dass eine auf den ersten Blick so europäisch scheinende Ausrichtung wie die der Konkreten Poesie durchaus über ,Wurzeln“ verfügt, die nach Lateinamerika gerichtet sind. Letztlich verweisen sie nicht nur auf brasilianische Avantgardisten der dreißiger und vierziger Jahre, sondern weiter zurück auf den Chilenen Vicente Huidobro, dessen avantgardistische Anfänge auf die Zeit der Futuristen zurückgehen. Vor allem die Arbeiten von Klaus Müller-Bergh haben im Verbund mit Gilberto Mendonça Teles eine weite und zugleich detaillierte Landkarte der Avantgarden in den verschiedenen Teilregionen Lateinamerikas entfaltet; diese gut dokumentierten Studien sollen als Grundlage für die nachfolgenden Untersuchungen immer wieder herangezogen werden. ${ }^{5}$

5 Vgl. die allgemeinen Überlegungen von Müller-Bergh, Klaus / Mendonça Teles, Gilberto: Los puntos cardinales de las vanguardias latinoamericanas. In: Gunia, Inke / Niemeyer, Katharina / Schlickers, Sabein / Paschen, Hans (Hg.): La modernidad revis(it)ada. Literatura y cultura latinoamericanas de los siglos XIX y XX. Estudios en homenaje a Klaus Meyer-Minnemann. Berlin: Edition tranvia - Verlag Walter Frey 2000, S. 241-251; dies. (Hg.): Vanguardia latinoamericana. Historia, crítica y documentos. Tomo I: México y América Central. Tomo II: Caribe, Antillas Mayores y Menores. Frankfurt am Main - Madrid: Vervuert - Iberoamericana 2000-2002; dies. (Hg.): Tomo III: Sudamérica. Area Andina Norte: Colombia - Venezuela. Madrid - Frankfurt am Main: Iberoamericana - Vervuert 2004; dies. (Hg.): Tomo IV: Sudamérica: Area Andina Centro: Ecuador - Peru - Bolivia. Madrid - Frankfurt am Main: Iberoamericana - Vervuert 2005; dies. 
Vicente Huidobro hat sich in der Tat schon früh mit der Problematik beschäftigt, dass die modernistischen Gedichtkonzeptionen noch immer der logischen, insbesondere kausalen Verknüpfung bei der Übermittlung von Inhalten anvertrauten, und damit einer Logik der Sprache und deren Ausdrucksfähigkeit. Ein quasi ,natürliches' Vertrauen, das der chilenische Dichter selbst nicht mehr aufzubringen vermochte. Vor dem Hintergrund zeitgenössischer Entwicklungen im Bereich der Malerei, insbesondere dem Kubismus mit seiner Collagetechnik, und der Lyrik, also der Formenentwicklung von Mallarmé bis Apollinaire, stellte sich Huidobro, der als Modernist begonnen hatte und gleichsam als „Modernista“ sozialisiert worden war, zunehmend der Frage, welche Ausdrucksmöglichkeiten über das herkömmliche Maß hinaus aktiviert werden könnten, um der Logik der gewöhnlichen Sprache zu entfliehen. Wir verstehen, dass sich Huidobro so mit Problemen herumschlug, wie sie gleichzeitig die italienischen Futuristen bearbeiteten, welche unter der Führung Marinettis bewusst in die Struktur gebundener Sprache eingriffen und bis zur Zerstörung von Alltagssprache avantgardistisch vorrückten.

Wir könnten aus der Beschäftigung mit den literarhistorischen Entwicklungen in den lateinamerikanischen Literaturen sagen, dass Vicente Huidobro schon früh aus chilenischer Perspektive den Versuch unternahm, dem abendländisch zentrierten europäischen Bewusstsein eine Dezentrierung dieser Konzeptionen des Okzidents entgegenzustellen. Die künstlerischen Möglichkeiten, die Huidobro auf seinem Arbeitsgebiet, der Dichtung, vorfand und Stück für Stück entwickelte, beziehen sich vor allem auf die graphische wie auch bildliche, ikonische Seite des poetischen Schreibens.

Man kann bei diesen frühen Kompositionen sehr wohl - wie dies Harald Wentzlaff-Eggebert tat - von Textbildern beziehungsweise Klangbildern sprechen, insoweit die lineare Abfolge sprachlicher Einheiten durchbrochen und andere Wahrnehmungsformen und Dechiffrierungstechniken auf den Plan gerufen wurden. Man darf derartige Verschränkungen von bildlicher und textueller Struktur gemäß der heutigen Literaturtheorie seit gut zwei Jahrzehnten als „Ikonotexte“ bezeichnen; ${ }^{6}$ und Sie werden sogleich vor Augen geführt bekommen, was es mit derartigen Ikonotexten auf sich hat. Wenn Sie übrigens weiterhin beim Ausdruck ,Textbilder‘ oder ähnlichen Wortfügungen bleiben möchten, dann

(Hg.): Tomo V: Sudamérica. Chile y países del Plata: Argentina - Paraguay - Uruguay. Frankfurt am Main - Madrid: Vervuert - Iberoamericana 2009.

6 Vgl. u. a. Wagner, Peter: Reading Iconotexts. From Swift to the French Revolution. London: Reaktion Books 1995; sowie ders. (Hg.): Icons - Texts - Iconotexts. Essays on Ekphrasis and Intermediality. Berlin - New York: Walter de Gruyter 1996. 
tun Sie dies ruhig: Mir kommt es in diesem Zusammenhang vor allem darauf an, dass Sie wissen, um was es sich handelt und welche Lektüreformen und Aneignungsweisen derartige Textbilder oder Ikonotexte voraussetzen. Denn es ist hochspannend, diese Wechselbeziehungen zwischen Bild- und Wortfügungen in ihrer Funktionsweise näher zu untersuchen.

Vicente Huidobros Technik ist dabei - avantgardistisch gesprochen - ganz in europäischer Avantgardetradition die des Schocks, eine Technik, die im Übrigen keineswegs avantgardistischen Ursprungs ist, sondern bereits von den Romantikern häufig verwendet wurde und sich unter dem Slogan „épater le bourgeois“ der Praxis des Bürgerschrecks zuordnen lässt. Wir könnten dies mit den russischen Formalisten wenden und davon sprechen, dass derartige Verfahren und künstlerisch-literarische Ausdrucksformen darauf abzielen, automatisierte Wahrnehmungsgewohnheiten und Lese- oder Sehweisen zu überraschen und außer Kraft zu setzen. Unsere Gewohnheiten werden mit den Mitteln der Kunst entautomatisiert, also bewusst gemacht oder in unser Bewusstsein gehoben, wodurch sie als Konventionen entlarvt werden. Mit anderen Worten: Was natürlich schien, erweist sich plötzlich als künstlich. Es handelt sich um ein Verfahren, wie es die Avantgarde der russischen Formalisten zeitgleich mit den europäischen Avantgardekünstlern mehrfach sehr überzeugend unter dem Stichwort der künstlerischen Verfahren (,priem“) abgehandelt hat. Auch das Verfahren der Entautomatisierung ist eines, das die historischen Avantgarden künstlerisch erstmals durchdacht haben, das aber im weiteren Verlauf der Literatur- und Kunstgeschichte auch weit jenseits der Avantgarden und Neo-Avantgarden Anwendung fand.

Was für literarische Folgen zeitigt ein derartiges Verfahren bei Vicente Huidobro? Ich möchte Ihnen gerne ein erstes Beispiel vor Augen führen, das freilich bereits aus seiner französischen Zeit, also nach seiner Übersiedelung nach Europa, stammt. Denn Huidobro war nicht nur als lateinamerikanischer Literat und Künstler in Paris, er war auch ein Französisch schreibender Pariser Künstler, wenn Sie so wollen. Sehen wir uns also einen ersten Ikonotext des Künstlers Vicente Huidobro aus dem Pariser Jahr 1921/2 mit dem Titel Moulin einmal genauer an!

Sie sehen schon auf den ersten Blick, dass wir hier nicht mehr mit unserer normalen Textanordnung auf dem weißen Blatt Papier auskommen, sondern eine andere Wiedergabeform wählen müssen. Dies hat übrigens dazu geführt, dass manche Ausgaben auf ein solches Gedicht wie Moulin ganz verzichten zu können glaubten. Sie können daran erkennen, wie sehr auch nach den avantgardistischen Klang- und Bildexperimenten unsere Gutenberg-Galaxis (wie Marshall McLuhan sagen würde) doch recht unverändert, jedenfalls an traditionellen Schrifttexten orientiert geblieben ist. Wenden wir uns nun aber diesem zweifellos herausragenden Ikonotext der historischen Avantgarden zu! 


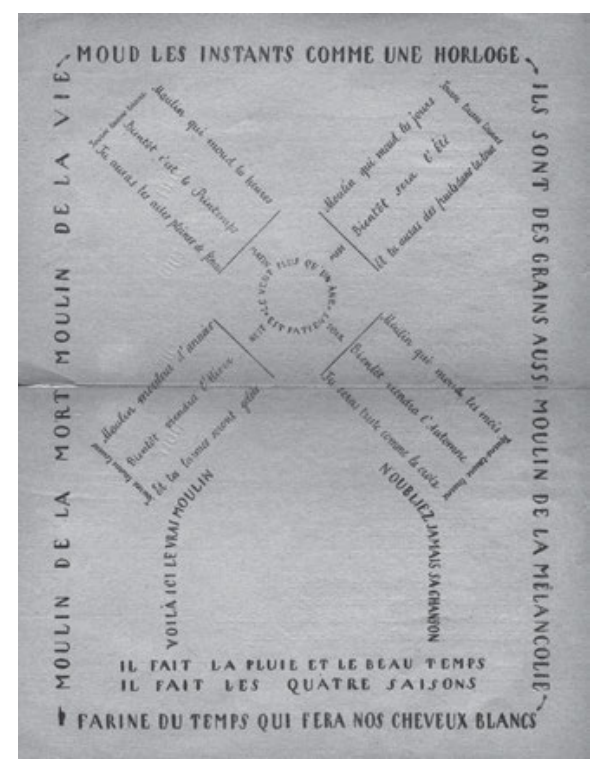

Abb. 57: Vicente Huidobro: Moulin, 1921-1922.

,Eigentlich' handelt es sich um ein Gedicht in klassischer Strophenform, die in ihrer Verteilung auf dem weißen Blatt Papier im Grunde nicht ins Bewusstsein dringen würde, wenn dieser gleichsam ,natürlichen' Form nicht eine andere graphische Lösung gegenübergestellt sein würde: die Anordnung in Form einer Windmühle, welche übrigens von Robert Delaunay entworfen wurde. Wir haben es bei diesem Gedicht in seiner Anordnung ohne Zweifel mit dem Verfahren der Entautomatisierung zu tun, eine Tatsache, die freilich übersehen wird, konzentriert sich doch in den meisten Untersuchungen das Hauptaugenmerk auf die faszinierendere, in gewisser Weise attraktivere Form des Ikonotexts. Es ist aber gerade die Differenz, die als solche schon thematisiert und inszeniert wird, ohne dass es einen Bruch zwischen der traditionellen und der avantgardistischen Form geben müsste. Auf dieser Ebene können wir also keinen Bruch konstatieren, durch den der Dichter mit der ,alten', traditionellen Form gebrochen hätte.

Lassen wir die Frage der Sprache, also die Verwendung des Französischen, außen vor! Vicente Huidobro griff auf das Französische zurück, weil er sich seit 1916 in Paris lebend - an ein französisches Publikum wandte und daher die Sprache der Besucher einer Ausstellung in einem Pariser Theater, dem Théâtre Edouard VII, wählte, die seine „poèmes peints“, seine „gemalten Gedichte“, sehen sollten. Doch auch hier stand die Wirkung des Schocks im Vordergrund. Vicente Huidobro musste dies mit der Tatsache bezahlen, dass die Ausstellung aufgrund des Protests zahlreicher Besucher nach wenigen Tagen schon wieder geschlossen werden musste und leider auch einige Gedichte Huidobros gänzlich 
verloren gingen. Es handelte sich wahrlich um ein engagiertes Publikum, das aktiv gegen diese Art von Kunst vorging - bis hin zu jenem Schauspieler in einem Theaterstück von Oliverio Girondo, der sich weigerte, das Publikum als ,dumm zu beschimpfen - aus Angst vor den Konsequenzen.

Denn bei den europäischen Avantgarden gehörte es zu den zentralen Verfahren, das Publikum zu schockieren, folglich als ,Bürgerschreck aufzutreten und aufzutrumpfen. Das „épater le bourgeois“ der Romantiker und Spätromantiker ist den Avantgardisten also geläufig, ja sie brauchen es bisweilen notwendig, um einen Gegenkanon künstlerischer Ausdrucksformen abzugrenzen. Dass diese Abgrenzung von ,traditionellen' Kunst- und Literaturformen innerhalb des eigenen Werkes nicht ganz so radikal wie oftmals inszeniert vonstattenging, zeigt - wie wir gleich sehen werden - ein Blick hinter die Kulissen des Gedichts, also in seine intratextuelle literarisch-künstlerische Vorgeschichte.

Huidobros Gedicht Moulin ist im Programm der Ausstellung, behandelt jedoch einen Gegenstand - die Windmühle -, der sich leitmotivisch im gesamten Werk Vicente Huidobros finden lässt: ein Bild, das den Dichter verfolgt und immer wieder beschäftigt zu haben scheint. Das Gedicht besteht aus einer Eingangszeile und 6 regelmäßigen, partiell reimenden vierzeiligen Strophen. Die Eingangszeile finden Sie wieder als Drehachse im Zentrum des Ikonotexts oder, wenn Sie so wollen, im Zentrum der Achse der Windmühlenflügel. Ein zusätzliches optisches Element war bereits den traditionell angeordneten Strophen insoweit zugeordnet worden, als für vier der sechs Strophen sich die Bezeichnungen „Matin“, „Midi“, „Soir“ und „Nuit“ (also Morgen, Mittag, Abend und Nacht) finden lassen. Sie bilden gleichsam den Tageszyklus, einen Kreis, der die zentrale Achse des Ikonotexts mit den einzelnen Windmühlenflügeln verbindet. Diese Flügel sind durch die jeweiligen Strophen gebildet und zu einem Kreis angeordnet, welcher die zyklische Wiederholung andeutet.

Die Kreisstruktur, welche in der Linearität des Strophengedichts nur auf einer höheren Abstraktionsebene eingefangen werden könnte, wird im Ikonotext visualisiert, keineswegs aber illustriert. Bei einem Ikonotext im engeren Sinne handelt es sich nicht um Text-Bild-Beziehungen, die wir in irgendeiner Weise als wechselseitige Illustrationen verstehen könnten. Das Bild illustriert nicht den Text, so wie der Text auch nicht das Bild illustriert oder erläutert. Ein Ikonotext im engeren Sinne ist vielmehr ein nicht mehr aufteilbares Ganzes, in welchem sich Text und Bild so sehr durchdringen, dass eine Zerstörung des gesamten Kunstwerks erfolgen müsste, würden wir einen Teil davon, sei es nun Bild oder Text, verschwinden lassen. Dass es sich in diesem Gedicht um eine Doppelstruktur handelt, was bei den anderen Beispielen der Ausstellung offensichtlich nicht der Fall war, spricht nicht dagegen, sondern macht nur auf den Sinnverlust aufmerksam und mehr noch auf den ästhetischen Verlust, den das Weglassen der visuellen Dimension 
verursachen würde. Ikonotexte sind symbiotische Gesamtkunstwerke: Text und Bild leben zusammen.

Die Kreisstruktur des Gedichts ist als Raumfigur selbstverständlich in die Zeitstruktur eingebunden. Wir können in unserer Kultur die Zeit nicht vom Raum trennen: Nicht umsonst sprechen wir von Zeit-Raum und Raum-Zeit. Dabei ist die Kreisstruktur als Zeitkonzeption der Wiederkehr eines Zeitablaufs zugeordnet, ganz so wie bei der zyklischen Abfolge der verschiedenen Teile eines Tages und einer Nacht. Insgesamt handelt es sich, von der Eingangszeile abgesehen, um vierundzwanzig Verse, was der Struktur der vierundzwanzig Stunden entspricht. Um sie drehen zu lassen, ist konstruktiv eine zusätzliche Zentralachse eingefügt, eben der Eingangsvers.

Die Mühle mahlt auch bereits im ersten Vers die Stunden, so dass dieses Element gleichsam visualisiert wird im Textbild oder, wie wir besser sagen sollten, im Bildtext oder ikonotextuellen Bildgedicht. Die vier hervorgehobenen Strophen sind parallel aufgebaut und drehen sich im wörtlichen Sinne um die Stunden, die Tage, die Monate und die Jahre, während zugleich Frühling, Sommer, Herbst und Winter die Kreisstruktur unterstreichen. Es ist der Zyklus der Zeit, der Natur und des Lebens.

Wir haben somit im Grunde zwei Zeitstrukturen, die miteinander verbunden sind. Sie haben prinzipiell bereits die beiden Anordnungsmechanismen des Gedichts im Raum kennengelernt: zum einen die Kreisstruktur des sich Wiederholens (der Tageszeiten oder der Jahreszeiten), zum anderen aber auch die Linearität bei den Stunden, den Tagen, den Jahren und - vergessen wir dies nicht - bei der sequentiellen Abfolge der Strophen selbst. Unsere Lektüre, unser Lesen ist prinzipiell linear. Die Resemantisierung der Windmühlenflügel folgt diesem Schema, insoweit es sich zum einen um eine zirkuläre Struktur handelt, zum anderen aber in der dritten Strophe, dem Abend oder auch dem Herbst, um ein Kreuz dreht, das als Sonnensymbol zwar gedeutet werden kann (und in der baskischen Svastika oder dem germanischen Hakenkreuz ja in der Tat auch zyklische Bedeutung als Sonnensymbol gewinnt), hier aber zweifellos als die Verbindung und Überkreuzung zweier linearer Gebilde, zweier Linien verstanden werden kann. Das Bild semantisiert den Text auf ebenso starke Weise wie umgekehrt: Es ist unmöglich, Bild und Text in diesem Ikonotext voneinander zu trennen.

Die wahre Mühle, so lernen wir zu Beginn der fünften Strophe, ist diese zeitliche Bewegung, die hier fixiert wird. Nicht umsonst bildet die fünfte Strophe das Mühlenhaus und vielleicht zwei Treppenstufen, die zu ihm führen, bildlich ab. Ganz traditionell und konventionell führen diese zeitlichen Allegorisierungen zur Vergegenwärtigung der vorübereilenden Zeit, die uns zum Tode führt, die folglich - mit Heidegger gesprochen - eine Zeit zum Tode ist. Dies konkretisiert sich 
in der sechsten Strophe, welche gleichsam den philosophischen (und reichlich konventionellen) Rahmen des Gedichts bildet und daher ganz zutreffend den Rahmen auch des Ikonotexts bestimmen muss. In diesem Rund der Schlussstrophe wird die Grundopposition Leben versus Tod evoziert und zugleich in eine kreisende Bewegung überführt, welche im Ikonotext an drei Seiten durch Pfeile signalisiert wird, an der vierten Seite jedoch zum Stehen kommt. Auf diese Weise blendet der Moulin die Dimension des Todes ein, das Zu-einem-Stillstand-Kommen der Zeit, des Lebens und Lebenswissens des Individuums wie der Gruppe: „Unsere Haare werden weiß“, auch dies erneut eine letzte Beziehung zum Mehl, das in der Windmühle gemahlen wird und uns einerseits Lebensmittel und damit Mittel zum Leben, andererseits aber das Zeichen des kommenden Todes ist. Innerhalb dieser semantischen Opposition von Leben und Tod, aber auch in dieser semantischen (und existenziellen) Ambivalenz bewegt sich das faszinierende Bildgedicht Vicente Huidobros.

All dies mag uns heute nicht sonderlich revolutionär vorkommen; und doch war es dies sehr wohl für die damaligen Zeitgenossen. Der chilenische Avantgardist hantiert in diesem Gedicht wie auch in anderen seiner Texte mit wirklichen Experimenten, die versuchen, sprachliche Ausdrucksformen an der Grenze des Sagbaren, des Semantischen, aufzuzeigen und künstlerisch-literarisch auszuprobieren. Führen wir uns nochmals vor Augen: Das Problem der Avantgarde - und zwar jeder Avantgarde - besteht darin, wie ihr Name schon sagt, dass sie die künstlerische oder politische und ästhetische Vorhut dieser Bewegung sein muss, also niemals zum Stillstand kommen darf. Immer wieder erweitert sie die Grenzen des Eigenen auf Kosten des Fremden (oder des Gegners, der feindlichen Tradition und bürgerlichen Konvention). Der vektorielle Bewegungspfeil ist der Avantgarde eingeschrieben.

Auch ein Vicente Huidobro musste immer weitergehen, um die avantgardistische Spannung seines Schaffens aufrechtzuerhalten - denken wir nur an den siebten Gesang in seinem Gedicht Altazor, das sich an der Grenze des Klanglichen, befreit vom Semantischen, vom Sinn-Machenden, von jeglicher stringenter Logik bewegt. Noch sind es zehn Jahre bis zur Veröffentlichung von Altazor und seines apokalyptischen siebten Gesangs, in welchem das Phonetische die Textualität nicht nur beherrscht, sondern schon - wenn auch nur ,fast" - ausfüllt. Dies ist der avantgardistische Weg zum A-Logischen, zum A-Kausalen, zum A-Semantischen, den Huidobro in seinem dezentrierenden Schreiben noch vor sich hat. In seinem lyrischen Ikonotext Moulin dreht sich noch alles um eine mehr oder minder feste existenzielle Achse.

Und doch war der Weg weit vom hispanoamerikanischen Modernismo zu avantgardistischen Experimenten, die wie in diesem Gedicht die Beziehung zwischen einem lebensweltlichen Gegenstand (einer Mühle) und dessen über- 
tragener Bedeutung wahrhaftig ins Bild (und einen Bildtext) setzen. Es ist ein sprachlich geformtes Sinn-Bild und komplexes Symbol, das - gerade weil es mit den Formen experimentiert - nicht allzu weit gehen darf im Bereich der schieren Inhalte, um überhaupt noch verständlich zu sein. Ist aber erst einmal die Technik, das künstlerische Verfahren, von der Leserschaft erfasst, so bedürfen diese Leserinnen und Leser nun anderer künstlerischer Kitzel, um zum Nachdenken angeregt und über Grenzen gebracht zu werden.

Sie sehen es unmittelbar ein: Eben dies ist die ,Mühle“ der Avantgarde und der Avantgardisten, die letztlich unter einem unerhört verschärften Originalitätsgebot stehen. Zugleich greifen sie aber auf das Lebensweltliche zurück, um dies wie die Readymades eines Marcel Duchamp, die noch in Enrique Vila-Matas' Erzähltexten eine wichtige Rolle spielen - auf die Institution Kunst oder Literatur zu beziehen und letztlich die Wahrnehmung dieser Institution selbst zu thematisieren, zu problematisieren, zu öffnen und zumindest utopisch auch zu überwinden. Gewiss, wir wissen es heute besser: Die Institution Kunst oder Literatur wird so mitnichten aus der Welt geschafft, sondern nur um eine neue Schraubenumdrehung fester gedreht. Denn die Kunst vereinnahmt ihre Infragestellung als Kunst und macht sie eben dadurch wieder marktgängig: Dies ist die Lehre der historischen Avantgarden, welche die Neo-Avantgarden in ihr Kalkül miteinbeziehen und theoretisch wie künstlerisch berücksichtigen mussten. Doch mit dem Gedicht Huidobros haben wir ein wichtiges Verfahren (,priem“ für die russischen Formalisten) entdeckt, wie es beispielsweise in der Konkreten Poesie vorherrscht: die Visualisierung des Bedeutungsgehalts eines Wortes. Genau dahin führte der bereits erwähnte lange Weg Huidobros vom Modernisten zum entschlossenen Avantgardisten: Es war ein mühseliger Weg, und doch scheint er Huidobro als Künstler-Literat Flügel verliehen zu haben.

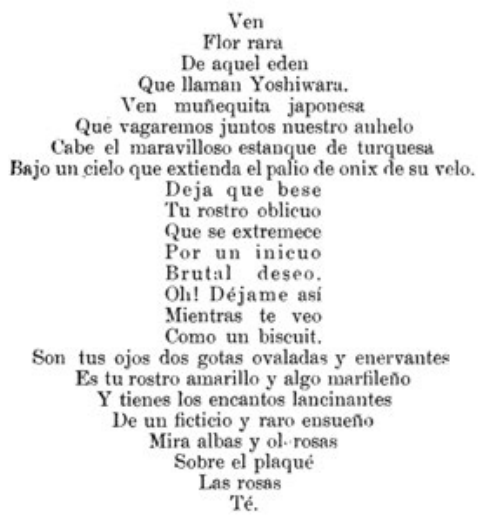

Abb. 58: Vicente Huidobro: Nipona, 1913. 
Den Übergang vom Modernismo zur „Vanguardia“ zeigen - oder visualisieren - sehr überzeugend jene frühen Textbilder oder Ikonotexte, die noch vor Huidobros Paris-Aufenthalt entstanden und in spanischer Sprache verfasst wurden. In seinen 1913 erschienenen Canciones en la noche finden sich vier Ikonotexte, von denen ich Ihnen zwei Beispiele kurz vorführen möchte.

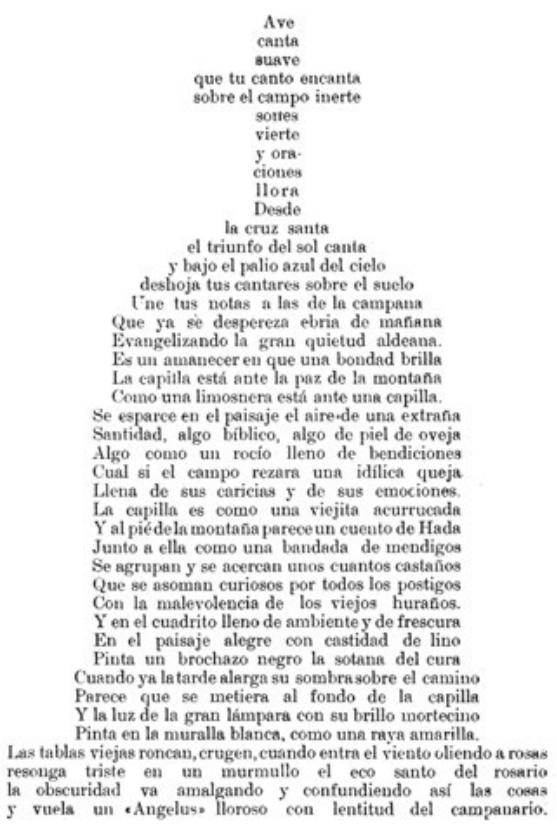

Abb. 59: Vicente Huidobro: La Capilla Aldeana, 1913.

Die beiden Gedichte weisen unterschiedliche Charakteristika auf. Das Gedicht Nipona ist ein Ideogramm, bei dem der poetische Text eine geometrische, aus einfachen Formen zusammengesetzte komplexere Form ausfüllt. Die einzelnen Zeilen oder Verse sind untereinander durch Kreuzreime verbunden, und dies unabhängig von der Silbenzahl, die zwischen einer Silbe und sechzehn Silben variiert. Die Lexeme dieser ,japonerías“ erinnern - vor allem in der ersten Strophe oder dem oberen Dreieck - unverkennbar nicht nur von der Thematik, sondern auch von ihrer Topik an die Sprache des Modernismo mit ihrer Farben- und Symbolwelt sowie der Freude am Ausgesuchten, Exquisiten, Exotischen.

Die Abwendung vom Modernistischen jedoch findet sich in der Anordnung der Gedichtzeilen zu ungewöhnlichen Formen. Eine gewisse Brüskierung des Modernismo bedeutet aber vor allem die semantische Überschreitung, wenn etwa plötzlich von „Brutal deseo“ oder auch vom „biscuit“ die Rede ist, wobei sich auf dem Gebiet des Körperlichen und Sexuellen Vergleichbares mitunter schon bei 
Rubén Darío - in seinem Gedicht Leda beispielsweise - finden lässt. Sie bemerken, dass es gar nicht so einfach ist, eine trennscharfe Grenze zum Modernismo anzugeben.

In Nipona beobachten wir ein poetisches Zusammenwirken des Optischen mit Rhythmus und Reim. Durchaus modernistisch ist die Orientalisierung des Gegenstandes in Verbindung mit der Projektion männlicher Phantasien auf eine orientalische Frau, die zum gehandelten Objekt in dieser lyrischen Inszenierung wird. Vergessen wir dabei nicht, dass in der zeitgenössischen Kunst immer stärker die Perfektion japanischer Haikus hervorgehoben wurde, die in ihrer fest gefügten Form bei gleichzeitig scheinbarer logischer Sprunghaftigkeit die westlichen Dichterinnen und Dichter gerade zu Beginn des 20. Jahrhunderts - und natürlich auch bis heute - in ihren Bann zogen.

Gleichviel, ob Chinoiserie oder Japonerie: Thematisch ist dieses Gedicht Nipona noch durchaus im Orbit des Modernismo oder wäre in gewisser - allerdings nicht kanonischer - Weise noch nach hispanoamerikanischer Terminologie als postmodernistisch $\mathrm{zu}$ bezeichnen. Im Vordergrund steht noch immer das Sprachmaterial selbst, eine Tatsache, die - wie wir sahen - in Moulin bereits nachhaltig relativiert ist und einem Ineinanderwirken der verschiedenen künstlerischen Ausdrucksformen Platz gemacht hat.

Betrachten wir Huidobros Ikonotext La Capilla Aldeana, dann sehen wir auf den ersten Blick, dass - ähnlich wie in Moulin der im Titel angekündigte Gegenstand in ein Schrift-Bild verwandelt und als Kontur gezeichnet ist: Es handelt sich also um ein Piktogramm. Auch in diesem Gedicht sind die sprachlichen Signifikanzen und differentiellen Momente der traditionellen Gedichtform beibehalten, ist beispielsweise der Reim doch auch hier vorhanden. Diese Beibehaltung des Reims bei verschiedenster Silbenzahl erlaubt eine Stärkung der Bewusstmachung der künstlerischen Form, die mehrfach selbstreferentiell ist. Denn nicht nur ist der Titel in die Konturen einer Dorfkapelle gefasst, auch das ,heilige Kreuz' selbst ist durch den Vers „la cruz santa“ am Fuße des Piktogramms des Kreuzes gleich mehrfach ästhetisch zum Ausdruck gebracht. Das Spiel mit dem „Ave“, das vom „suave“ echoartig wiederaufgenommen wird, führt die Dimension des Akustischen ein, macht den Ikonotext damit auch zu einem Phonotext. Die Vieldeutigkeit des verwendeten sprachlichen Materials wird artistisch in den Mittelpunkt gerückt, wobei bemerkenswert ist, dass der durch „Ave“ mitbedeutete Vogel nicht graphisch repräsentiert wird. Ist er (etwa bei der Annäherung des Priesters mit seiner schwarzen Soutane) schon wieder weggeflogen?

Die traditionelle Gedichtform erweist sich ebenfalls daran, dass das experimentelle Element gegenüber dem späteren Moulin noch deutlich zurückgenommen erscheint und zugleich die Linearität der Wahrnehmung des Gedichts in keiner Weise eingeschränkt ist: Wir lesen das Gedicht von Anfang bis Ende, 
von oben bis unten, lückenlos durch. Die narrativen Handlungselemente treten deutlich hervor, ihre Logik und Kausalität ist im Grunde ungebrochen. In der Tat ist die Übereinstimmung von Titel, Konturen und Inhalt im Sinne einer klaren Referentialisierbarkeit gegeben, so dass das eigentlich Artistische allein darin zu liegen scheint, wie es dem Künstler gelang, sein Sprachmaterial so anzupassen, dass es der vorgegebenen Form einer Kapelle gerecht werden konnte. Dies freilich ist ein eher handwerklicher denn künstlerischer Aspekt. Die sprachliche Aussage dominiert noch immer, ähnlich wie in den bekannten barocken Bildgedichten, in deren lange Traditionslinie sich dieser Ikonotext fraglos einschreibt.

Huidobros Kunst zeigt sich in diesen Gedichten jener Guillaume Apollinaires verwandt, der 1914 mit Moi aussi je suis peintre ähnliche Versuche der Visualisierung lyrischer Texte unternahm. Apollinaire war zweifellos ein wichtiges Bindeglied hinüber zur literarischen Avantgarde, ein Dichter, der für viele als Orientierungspunkt diente und gerade deshalb von bestimmten Avantgardisten - wie wir sahen - vehement angegriffen wurde. Vicente Huidobros eigentliche literarästhetische Leistung freilich wird sich erst im Umfeld seiner Auseinandersetzung mit den französischen Lyrikern und Künstlern mit ihren breiten Ausdrucksmöglichkeiten entwickeln und dabei zu jener Formel des Creacionismo finden, die er - unabhängig und zusammen mit seinem früheren Freund und späteren Feind Pierre Reverdy - entwickeln sollte. Dabei wandte er sich gegen einen simplen Mimetismus, gegen ein einfaches Abspiegeln der Dinge und stellte einer so verstandenen Mimesis den schöpferischen Akt, das Erschaffen der Dinge durch den Dichter-Demiurgen, durch den Poeten dieser „Visuellen Poesie“, entgegen. Dass dies keineswegs im Sinne eines A-Mimetismus der Fall sein musste, hat Karin Hopfe in einer 1996 erschienenen Dissertation über die Lyrik Vicente Huidobros gezeigt. $^{7}$

Lassen Sie mich an dieser Stelle eine kleine europäische Rückblende einbauen! Denn die Entwicklung des Bildgedichtes ist ein für die historischen Avantgarden insgesamt so wichtiger Schritt, der dann auch die Entfaltung der Konkreten Poesie anregen sollte, dass wir noch einmal auf die Dimension des Graphischen anhand erster Experimentalversuche zu Beginn unseres Jahrhunderts zurückkommen sollten. Als jene Figur, die dem Experimentellen gerade in Hinblick auf die visuelle, die graphische Dimension des Gedichts zu einem frühen Zeitpunkt die vielleicht entscheidenden Erkenntnisse abringen konnte, darf wohl - auch wenn uns Vicente Huidobro in dieser Frage bestimmt nicht Recht geben würde - Guillaume Apollinaire gelten. Er war, wie wir sahen, nicht nur

7 Vgl. zur Bedeutung der Mimesis Hopfe, Karin: Vicente Huidobro, der Creacionismo und das Problem der Mimesis. Tübingen: Gunter Narr Verlag 1996. 
Bezugspunkt für die Futuristen, sondern viel mehr noch für die Dadaisten; und auch den französischen Surrealisten gab er entscheidende Impulse mit.

Guillaume Apollinaire, der eigentlich Wilhelm Apollinaris de Kostrowitzky hieß, wurde in Rom am 26. August 1880 als unehelicher Sohn einer polnischen Mutter und eines italienischen Vaters geboren. Er lebte vor allem in Paris, wo er sich früh mit Vorläufern der Avantgarde wie Alfred Jarry anfreundete und nach einem intensiven Leben als Dichter und Künstler am 9. November 1918 verstarb. Er erlitt 1915 an der Front im Ersten Weltkrieg eine Kopfverletzung und wurde wenige Tage vor Kriegsende, noch geschwächt, zu einem der zahlreichen Opfer der Spanischen Grippe, jener Pandemie, die zusammen mit dem Weltkrieg der vorangehenden Phase beschleunigter Globalisierung ein Ende setzte. Doch sein Schaffen ist bis heute nicht nur in Frankreich sehr lebendig geblieben.

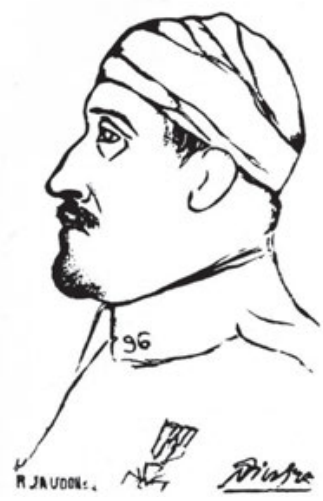

Abb. 60: Guillaume Apollinaire (Rom, 1880 - Paris, 1918).

Seine zu Lebzeiten bedeutendste Gedichtsammlung Alcools erschien 1913, doch seine kurz nach seinem Tod veröffentlichten Calligrammes waren Texte, deren Bedeutung in der typographischen Anlage lag und in denen Apollinaire mit der visuellen Kraft der graphischen Anordnung des Sprachmaterials experimentierte. Neben dieser semantischen Intensivierung des Wortmaterials arbeitete Apollinaire auch verstärkt mit Collagen und erneuerte die künstlerischen Ausdrucksmöglichkeiten seiner Zeit. Als Freund Jarrys wirkte er nachdrücklich auf die Entwicklung eines surrealistischen Theaters ein. Dass er zu seinem Broterwerb auch pornographische Romane verfasste, gehört $\mathrm{zu}$ dieser schillernden Persönlichkeit eines Dichters, den man in vielerlei Hinsicht Huidobros Creacionismo hätte zuordnen können, hätte er nicht in Paris gelebt und sich ausschließlich für das Französische entschieden.

Die visuelle Gestaltung von Gedichten geht zunächst von der simplen Tatsache aus, dass sich jedes Gedicht, in welcher Sprache es auch immer verfasst 
sein mag, in schriftlicher Form schon durch seine typographische Gestalt, durch seine „mise en page“, zu erkennen gibt. Daraus entspringt die Vorstellung, diesen Bereich experimentell auszutesten. Das lyrische Textbild erzeugt schon per se eine Simultaneität der Wahrnehmung, wie sie den Avantgardisten ins Auge stach oder stechen musste. Es ging nur darum, diese gleichsam ,natürliche‘ Wahrnehmung der typographischen Gestaltung eines Gedichts radikal zu entautomatisieren.

Ein verändertes Textbild provoziert eine Art Unmittelbarkeit, erschwert aber zugleich auch die Wahrnehmung durch eine an Konventionen gewöhnte Leserschaft. Würde man die verschiedenen literarischen Gattungen nicht nach ihrem Umfang, nach inhaltlichen Kriterien oder anderen Aspekten, sondern nach der jeweils erforderlichen oder praktizierten Lesegeschwindigkeit unterteilen, so wären Gedichte ganz zweifellos jene literarische Form, bei der die Lesegeschwindigkeit am niedrigsten liegen dürfte. Das Bildgedicht nun reduziert diese Geschwindigkeit noch einmal sehr, da der Unmittelbarkeit einer ersten, quasisimultanen Wahrnehmung eine hernach durchaus erschwerte schriftsprachliche Wahrnehmung folgt. Die inter- und transmediale Verschränkung von Text und Bild im Ikonotext verlangsamt die Wahrnehmungs- und Lesegeschwindigkeit zusätzlich: Dies gilt es zu berücksichtigen, wenn man sich poetischen Ikonotexten annähert.

Guillaume Apollinaire experimentierte seit 1911 mit verschiedenen Formen der Verschränkung von Text und Bild, also mit unterschiedlichen Formen poetischer Ikonotextualität. In der Tat haben wir es in diesen Fällen mit einer inter- beziehungsweise transmedialen Verstärkung sinnbildender und sinnmultiplizierender, sich wechselseitig verstärkender semantischer Prozesse zu tun: intermedial, wenn sich ein Dialog zwischen zwei medialen Ebenen - etwa Bild und Schrift - ergibt; transmedial, wenn die Querung unterschiedlicher Ebenen etwa zwischen Sprache, Klang und Bild hin- und herläuft.

Der erschwerte Übergang vom Schauen zum Lesen betrifft bei dem nun folgenden Gedicht Apollinaires, mit dem wir uns nur kurz beschäftigen können, vor allem die Anordnung der Leserichtung, wenn auch nicht die vorherrschende, von links nach rechts horizontal verlaufende Leserichtung insgesamt. Denn wir müssen nach der ersten Betrachtung in der Tat notwendig das Gedicht drehen, um es lesen zu können. Dann aber sehen wir die folgenden Verse des Gedichts Il pleut aus Apollinaires Calligrammes in seiner überzeugenden graphischen Gestalt und verstehen vielleicht besser, warum der Dichter seine Texte ursprünglich als „,idéogrammes lyriques“ bezeichnete. Das frühe Interesse Apollinaires für verschiedene Schriftarten und insbesondere für die chinesische Schrift ist noch erahnbar. Das Gedicht Il pleut aus dem Jahre 1914 besteht aus insgesamt fünf Versen, wobei die Worte dieser Verse nach futuristischer Manier gleichsam ,frei ${ }^{\star}$ oder ,befreit' sind: 


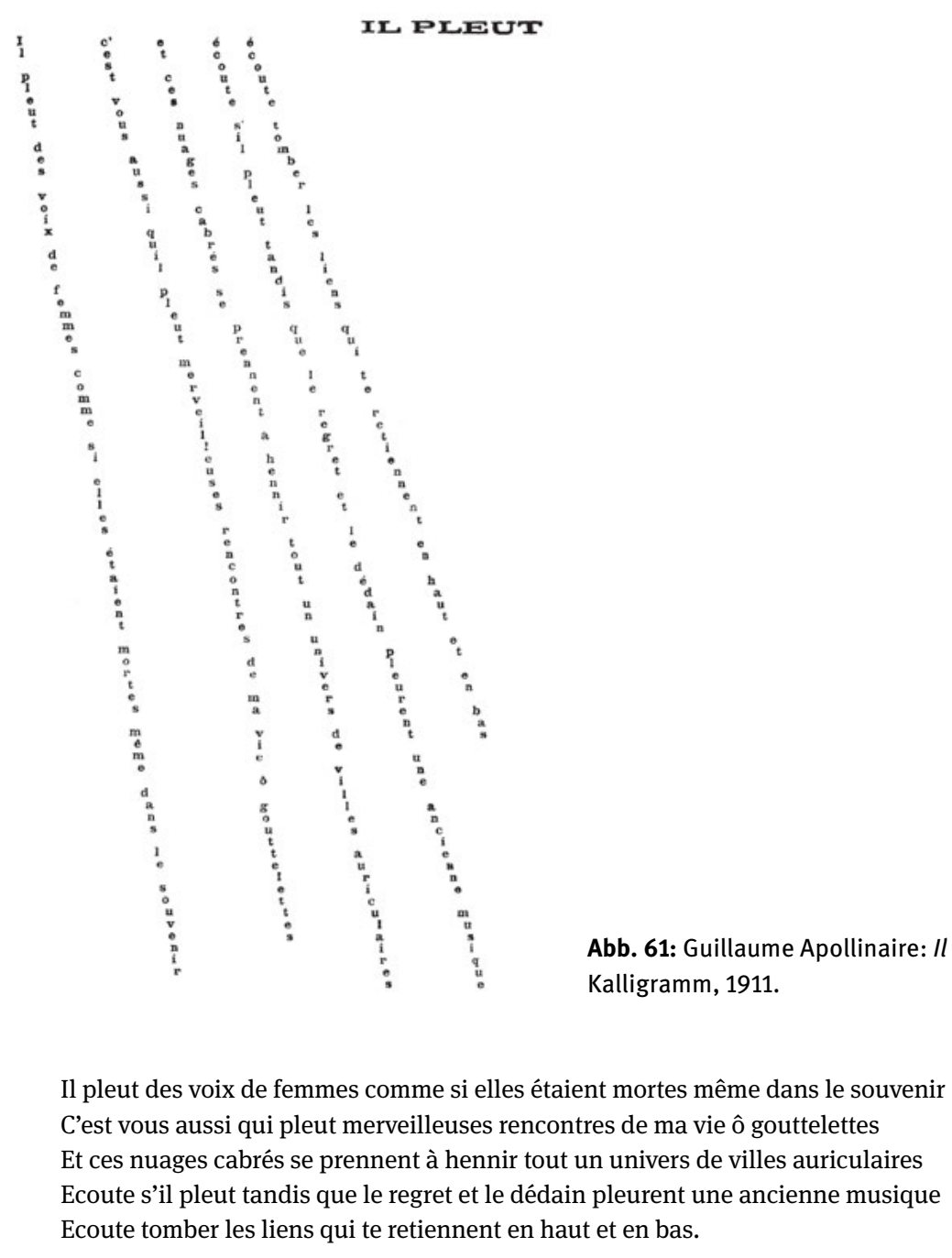

Das ikonotextuelle Bildgedicht ist vertikal und nicht horizontal angeordnet. Es bildet gleichsam Wortschnüre, die ohne Interpunktion die Worte aneinanderreihen und auch manche für das Französische so wichtige Apostrophes unterdrücken. Die Verse folgen keinem Reimschema und stellen daher „,vers libres“ dar. Die verlangsamte Geschwindigkeit der Wahrnehmung des Gedichts erhöht ganz zweifellos die Semantizität der zu entziffernden Regenschnüre, von denen der letzte, der im Übrigen genau das ,Unten` thematisiert, das Unten des Textes, gleichsam das Auftreffen der Regenschnüre auf den Boden, nicht mehr erreicht. So gibt es eine Art schwebenden Abschluss. 
Apollinaires lyrische Kreation besitzt eine einfache Grundstruktur: Die beiden ersten Verse des Gedichts evozieren das Gedächtnis des Dichters und die Vergangenheit, sind also ganz der Erinnerung gewidmet. Im dritten Vers, der eine zentrale Stellung einnimmt, beobachten wir einen Übergang, der in die Kontemplation des Himmels eingebettet ist. In den beiden abschließenden Versen konzentriert sich das Gedicht auf die Wahrnehmung, auf das Hören und die Musik. Es ist ganz zweifellos so, dass sich die vertikale Anordnung auch als ein Absteigen, ein Hinuntersteigen in die Vergangenheit deuten lässt, wobei das Schweben des letzten Verses über dem Boden eine eigentümliche Spannung erzeugt. Die Vertikalität der Regenschnüre versinnbildlichen eine „descente“ in die Bereiche der Unterwelt, des Unbewussten.

Beim Hinabsteigen in die Vergangenheit sind die Frauenstimmen, erst einmal ganz unten angekommen, im „souvenir“ platziert, in der Erinnerung des Endes der ersten Regenwortschnur. Mag sein, dass damit autobiographisch das Ende einer Liebesbeziehung Apollinaires mitgemeint ist. Das Thema der Begegnung, ja der wunderbaren Zufallsbegegnung, ist im zweiten Vers thematisiert, so wie auch die Begegnung mit ganz bestimmten Regenschnüren selbstverständlich eine der zufälligen Art ist. Der Zusammenklang, die Musik der Regentropfen, kurz: die Phonotextualität spielt eine wichtige Rolle: Die Synästhesie bildet eine zentrale Dimension dieses mit einer Stadtlandschaft verknüpften Gedichts, in welchem Bild, Schrift und Klang in den Regentropfen zusammenkommen. Auch das taktile Element ist vom ersten Vers an präsent.

Es regnet offenkundig auf eine Fläche in einer urbanen Landschaft, wobei die unterschiedlich langen Regenschnüre auch eine Art Dreidimensionalität der Wahrnehmung erzeugen. Wichtig scheint mir bei diesem Bildgedicht die Tatsache, dass auf der inhaltlichen Ebene weniger das Visuelle als vielmehr das Akustische thematisiert wird. Dies beginnt mit den Stimmen der Frauen und setzt sich dann fort mit verschiedenen Geräuschen, auch dem Geräusch des Regens selbst, bis hin zum menschlich organisierten Klang, der Musik und damit der akustischen Kunst. So bestimmt das Hören in diesem Bildgedicht die eigentliche Fokalisierung der Wahrnehmungsprozesse, die gerade auch in der direkten Anrede der Leserschaft eingefordert werden. Die Aufforderung des Zuhörens ergeht im Medium des schriftlichen Textes, der auf die lautliche Sprache und Anrede des Dichters verweist.

Wir sollten an dieser Stelle nicht weiter in das Gedicht Il pleut eindringen, da wir für unsere Vorlesung bereits wichtige Erkenntnisse sammeln konnten. Die Lyrik von Guillaume Apollinaire aktiviert alle Sinne, spricht alle Sinne an und bringt sie miteinander in Verbindung. Sinn wird zu einer Dimension sinnlicher Erfahrung und nicht zu einer Folge logisch aufeinander aufbauender Syntagmen. Apollinaires Il pleut, das letzte seiner zweiten Sammlung Calligrammes, lotet auf 
herausragende Weise viele Möglichkeiten eines Bildgedichts aus, die in der Folge nicht zuletzt von den französischen Surrealisten und den Vertreterinnen der Konkreten Poesie, aber auch schon von Vicente Huidobro sehr kreativ umgesetzt worden sind. Lassen Sie uns nach diesem kurzen Abstecher wieder zurück zu diesem wichtigen Vorläufer und frühen Vertreter der Avantgarden in Lateinamerika zurückkehren, wenn auch sicherlich zu einem Vertreter, der gerade mit den Entwicklungen in Europa und insbesondere in Frankreich aufs Engste vertraut war.

Im Rahmen dieser Vorlesung ist es uns leider nicht möglich, der Theorie und vor allem der Praxis des Creacionismo in all ihren Einzelheiten nachzugehen und die zahlreichen Widersprüche und kreativen Missverständnisse im Schaffen des chilenischen Dichters aufzudecken. Es handelt sich bei Huidobros Creacionismo keineswegs nur um einen mehr der zahlreichen „Ismos“, welche die Jahrzehnte bis zur Mitte des Jahrhunderts bevölkern sollten, sondern um eine der einflussreichsten künstlerischen Strömungen eines der sicherlich herausragenden Dichter der Avantgarde in Lateinamerika. Der Creacionismo, der als Begriff möglicherweise als Schimpfwort nach einem Vortrag Huidobros entstand, hat dabei im engeren Sinne nie schulbildend, stets aber befruchtend auf die zeitgenössische Lyrik Hispanoamerikas und bis hinein in unsere Zeit impulsgebend gewirkt. Dass der Creacionismo aber nicht - wie häufiger zu lesen - auf eine Creatio ex nihilo setzte, sondern sich sehr wohl in existierende literarische und künstlerische Traditionen und Filiationen einschrieb und mit zahlreichen Modellen anderer Poetiken spielte, dürften unsere Überlegungen und die Beispiele von Ikonotexten in aller wünschenswerten Deutlichkeit gezeigt haben. Auch in diesem Sinne bildet diese Strömung nicht die Ausnahme, sondern vielmehr die Regel der Avantgarden in Lateinamerika.

Vicente Huidobro ist keineswegs nur von europäischen Koordinaten der Avantgarden her zu bestimmen, weist auch seine Lyrik und vielleicht mehr noch seine Strategie innerhalb des literarischen und intellektuellen Feldes auf die äußerst engen Beziehungen zur europäischen und insbesondere französischen Avantgarde hin. Von Beginn an standen in Lateinamerika andere Konzepte und Zielsetzungen der Avantgarden im Vordergrund; und es wäre sträflich sowie wissenschaftlich verantwortungslos, würde man sie in ihren Entwicklungen lediglich als ,Abweichung، von einem europäischen Vorbild oder Modell behandeln und abtun.

Zum freien Umgang mit den europäischen Ideen, Vorstellungen und Praktiken gehört insbesondere die gezielte Verwendung der in den Avantgarden so beliebten Textsorte des Manifests, dessen erstes Huidobro im Jahr 1914 verfasst hatte. Diesem ersten Manifest sollte noch eine lange Reihe weiterer folgen, die Huidobro später gesammelt (wenn auch nicht in vollständiger Reihung) heraus- 
gab. Gegenüber den ersten Manifesten der europäischen Avantgarden ist bei Huidobros avantgardistischen Aktivitäten mithin eine nur wenige Jahre umfassende ,Verspätung` zu beobachten, die aber nicht als rein zeitliche Verspätung zu begreifen ist, sondern mit dem Zeit-Raum und der spezifischen Situation Hispanoamerikas in Verbindung gebracht werden muss.

Vicente Huidobros erstes Manifest erschien kaum später als das Gründungsmanifest des italienischen Futurismus von 1909: Diese Publikation liegt durchaus noch im Bereich einer relativen Simultaneität. Vergleichen wir kurz mit der Situation in Europa: In Deutschland etwa datiert das erste futuristische Manifest in Form eines offenen Briefes von Alfred Döblin an Filippo Tommaso Marinetti auf das Jahr 1913; die ersten Manifeste in Frankreich stammen von 1912; das Intersektionistische Manifest von Fernando Pessoa erschien in Portugal 1915; Tristáns Futuristische Proklamation an die Spanier datiert auf 1910; die ersten russische Manifeste erschienen 1912. Wir können also wohlfundiert von einer relativen Gleichzeitigkeit und nicht länger von einer bedeutsamen ,Verspätung، sprechen.

Was aber beinhalteten diese Manifeste? Wir haben uns ja bereits zweimal in unserer Vorlesung ausführlicher mit der Textsorte des Manifests auseinandergesetzt. Wir sind dabei in guter Gesellschaft, beschäftigte sich doch Vicente Huidobro selbst mit dieser Art von Literatur recht intensiv. Er tat dies ebenso reflexiv wie selbstreflexiv, ebenso kritisch wie selbstkritisch: Er führte seine literarischen Untersuchungen beispielsweise in seinem Manifestes Manifeste durch, das ich Ihnen hier in deutscher Übersetzung vorlege und nicht in der 1925 ursprünglich auf Französisch publizierten Fassung eines gewissen Vincent Huidobro:

Nach den letzten, auf die Dichtung geschleuderten Manifesten habe ich meine eigenen neu gelesen und finde mich, mehr als zuvor, in meinen alten Theorien wieder.

Die Manifeste Tristan Tzaras liegen vor mir sowie drei surrealistische Manifeste und meine eigenen Aufsätze und Manifeste. Was ich zuerst feststelle, sind gewisse Dinge, die uns allen gemeinsam sind, eine logische Überschätzung der Dichtung und eine ebenso logische Geringschätzung des Realismus. Der Realismus in der gewöhnlichen Bedeutung des Wortes, d.h. der mehr oder weniger geschickten Beschreibung von Wahrheiten, die schon zuvor existieren, interessiert uns nicht und wir diskutieren diese nicht einmal, denn die Wahrheit der Kunst beginnt dort, wo jene des Lebens endet. Der Realismus hat in unserem Land kein Bürgerrecht.

Die dadaistischen Manifeste Tzaras sind zu seiner Zeit heftig diskutiert worden, dass es nicht der Mühe wert ist, darauf zurückzukommen. [...]

Nun aber entzieht diese Form des Schreibens, das die Feder unter dem Einfluss eines automatischen, dem Traum entsprungenen Diktats führt, dem Dichter und der Dichtung die ganze Kraft seines natürlichen Rauschs (natürlich bei Dichtern). D.h. sie entzieht ihm das tief verwurzelte Geheimnis von Ursprung und Ausführung, das gesamte Spiel der Wörterzusammenführung, ein bewusstes Spiel, selbst im Fieber des größten lyrischen Rausches, also die einzige Angelegenheit, die den Dichter begeistert. Wenn man mich des Augenblicks der Produktion, des wunderbaren Augenblicks des so übermäßig offenen Blickes, 
der das Universum füllt und wie eine Pumpe aufsaugt, berauben würde, des begeisternden Moments dieses Zusammenführungsspiels auf dem Papier, dieses Schachspiels gegen das Unendliche, des einzigen Augenblicks, der mich die Alltagsrealität vergessen lässt, dann würde ich Selbstmord begehen. [...]

Die Dichtung ist etwas viel Schwerwiegenderes, weit Großartigeres, sie entspringt unserem Überbewusstsein.

So wie ich es bei meinen Vorträgen in Buenos Aires, Madrid, Berlin, Stockholm und Paris, im Theater am Square Rapp im Januar 1922, gesagt habe: „Das kreationistische Gedicht entsteht allein aus einem Zustand des Überbewusstseins oder des dichterischen Rausches.“8

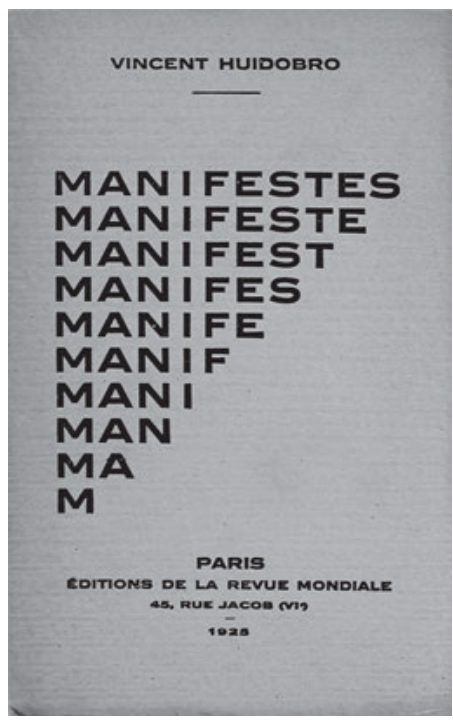

Abb. 62: Cover der Erstausgabe von Vicente Huidobros Manifestes Manifeste ..., 1925.

Wie Sie sehen, geht die selbstbewusst behauptete Kontinuität der eigenen schöpferischen Theoriebildung und der eigenen dichterischen Praxis von einer Lektüre anderer, gleichsam konkurrierender Manifeste, aber auch von den eigenen Proklamationen, Vorträgen und Reden aus, versucht jedoch zugleich, die Theorie des Creacionismo angesichts der zeitgenössisch dominant gewordenen Theoriebildungen und insbesondere der surrealistischen Praxis der „écriture automatique“ konsequent zu behaupten. Vicente Huidobro versteckt sich mit seinen Manifesten nicht gerade hinter den großen Manifesten der Futuristen oder der Dadaisten Europas. Er verweist auf seinen eigenen kreationistischen Ansatz.

8 Huidobro, Vicente: Manifeste Manifestes (1925). In: Asholt, Wolfgang / Fähnders, Walter (Hg.): Manifeste und Proklamationen, S. $354 \mathrm{ff}$. 
Dabei spricht er gegenläufig zu den Theorien Sigmund Freuds, auf welche die Surrealisten stark rekurrierten, von einem Überbewusstsein und hebt im Gegensatz zu Tendenzen, die zentrale Position des Subjekts im automatischen Schreiben aufzulösen, die Rolle des schöpferischen Poeten hervor. Dichtung und Rausch lassen uns nicht nur an die Surrealisten und ihre Aufwertung des Unbewussten denken, sondern zugleich auch erkennen, dass sich Vicente Huidobro sehr früh schon intensiv mit dem Vater der Psychoanalyse literarästhetisch auseinandersetzte und sehr persönliche künstlerische Konsequenzen aus dieser Beschäftigung zog. In seinen Überlegungen blieb er freilich Funktion und Rolle des großen Poeten verpflichtet, wobei für ihn das Produzieren von Lyrik - die verwendete Metaphorik zeigt es an - nach wie vor im Mittelpunkt des schöpferischen Prozesses steht.

Huidobro lehnt in seinem Manifest eine ganz bestimmte Form des Realismus $\mathrm{ab}$, jene nämlich, welche die Dinge der Realität in die Kunst transponiert und mimetisch direkt anzueignen bestrebt ist. Sein Augen-Blick des übermäßigen Blickes, der das Universum füllt und danach alles wie eine Pumpe in sich hineinsaugt, ist auf einer zweifachen Bewegung aufgebaut: Jenem gewaltigen Füllen des Universums durch den Dichter und Schöpfer und danach jenem Einatmen, Einsaugen der so gefüllten Welt - ein organischer Prozess von Ausatmung und Einatmung. Dies ist weit von einer kompletten Absage an die äußere Realität entfernt, sondern eine Abkehr von einer bestimmten Art und Weise ihrer Anverwandlung. Huidobros Verhältnis zur Mimesis bleibt spannend und geht keineswegs in Richtung eines dichterischen Hermetismus, wie dies bisweilen zu lesen ist.

Kreationistisch meint im Sinne des chilenischen Dichters also keine Creatio ex nihilo, wie sich auch hier zeigt, sondern vielmehr eine dialogale und mehr noch polylogische Form von Anverwandlung, in welcher der Dichter zugleich über den Rauschzustand und die Fähigkeit höchster kognitiver Wahrnehmung, zugleich über dunklen Traum und rationales Schachspiel verfügt und gebietet. Wir haben es nicht mit einer Auflösung des künstlerischen Subjekts, sondern eher mit dessen Überhöhung zu tun, was gewiss auch autobiographische beziehungsweise in der starken Persönlichkeit Huidobros verankerte Gründe besitzen kann.

Es sind folglich - um mit dem Begriff des Manifests etwas zu spielen - das Manifeste und das Latente, der Trauminhalt und eine bestimmte Schicht der Realität, welche Eingang in den Höhepunkt schöpferischen Tuns finden. Dass wir in diesem auf Französisch verfassten Manifest wie auch in den meisten anderen Manifesten Huidobros nichts zur spezifisch lateinamerikanischen Situation erfahren, sollte uns nicht beunruhigen, handelt es sich bei diesem Text doch um eine sehr gezielte Auseinandersetzung mit der französischen Avantgarde. Die Integration in die spezifisch lateinamerikanischen Kontexte und Filiationen blieben Huidobro sehr bewusst, hätten an dieser Stelle aber - angesichts eines noch bis 
in unsere Tage spürbaren Eurozentrismus in der Theoriebildung der Avantgarde den Effekt, die Wirkung auf eine europäische Leserschaft gestört. Im Übrigen wäre es, um erneut mit Jorge Luis Borges zu sprechen, gerade kein Zeichen einer wie auch immer deutbaren ,Lateinamerikanizität‘, wenn das Manifest nur so von vermeintlichen Lateinamerikanismen gefüllt gewesen wäre. Vicente Huidobro ging es nicht darum, Kamele in den Koran zu tragen.

Im Übrigen war der chilenische Dichter und Theoretiker innerhalb des europäischen Kontexts keineswegs der einzige, der sich Gedanken über die eigenen, vor allem aber die anderen Manifeste machte und diese publizierte. Auch der Dadaist Tristan Tzara, der in Huidobros Manifest Erwähnung fand, verfasste nicht nur Manifeste, sondern Meta-Manifeste: Manifeste über Manifeste also. Ein solches Meta-Manifest ist auch Huidobros Manifestes Manifeste und damit keineswegs ein Solitär. Es inszeniert den Dichter in seiner Produktion, zeigt eine Visualisierung des schöpferischen Aktes: Nicht umsonst dominiert gerade hier der offene Blick ins Universum, der Augenblick höchster optischer Wahr-Nehmung, wie sie so Huidobro - allein dem Dichter und somit dem herausragenden Individuum in seinem Kreationsprozess zukomme. Dass hiermit nicht bloß ein bestimmtes Lebensgefühl, sondern vor allem eine Hierarchie innerhalb der künstlerischen Tätigkeiten gemeint ist und durchgesetzt werden soll, versteht sich von selbst.

Auffällig ist bei diesem Manifest Huidobros sehr wohl, dass es - ganz im Gegensatz beispielsweise zu jenen der französischen Surrealisten - die Rezeptionsseite nahezu vollständig ausblendet, die Aneignung bestimmter Techniken noch nicht einmal erwähnt oder höchstens - wie im Falle der ,écriture automatique“ - mit Abfälligkeiten bedenkt, um allein dem schöpferischen, dem kreationistischen Gestus, dem Furor divinus des Dichter-Demiurgen, die volle Aufmerksamkeit zu schenken. Wie bei Rubén Darío, der in einem berühmten Gedicht von den Dichtern als „Torres de Dios“, als „Türmen Gottes“ sprach, wie im hispanoamerikanischen Modernismo bleibt die Rolle des Dichters als Weltenschöpfer unangetastet.

Diese einseitige Ausrichtung am herausragenden Dichter-Subjekt ist bewusst und gewollt. Nicht nur auf Grund der zum Teil klaren, eindeutigen Frontstellung gegenüber den europäischen Surrealisten, nicht nur auf Grund von Huidobros nietzscheanischen Überzeugungen, auf welche letztlich seine Stärkung der Rolle der Imagination und seine Konzeption des „superconsciente“, des „Überbewusstseins“, zurückgehen. Diese Position ist das Ergebnis einer sich im zweiten Jahrzehnt des zurückliegenden Jahrhunderts vollziehenden Bewusstwerdung Huidobros, die Konzepte der hispanoamerikanischen Modernisten als normgebende Vorgänger zu kritisieren, zu negieren und schließlich zu bekämpfen, auch wenn noch sehr viel Modernistisches in seinen Gedankengängen fortlebt. Dies war geradezu notwendig der Fall, muss man sich doch immer vergegenwärtigen, dass 
es Rubén Darío und damit der damals führende Modernist war, der bereits 1909 im Bonaerenser Periodikum La Nación die Argentinier und Lateinamerikaner insgesamt mit den neuen, beunruhigenden futuristischen Ideen aus dem Europa vor dem Ersten Weltkrieg bekanntmachte.

Im oben angeführten Manifest Huidobros ist des Weiteren die Kombinatorik wichtig, also das Schachspiel mit bereits vorhandenen Elementen: Die spezifische Fähigkeit des Dichters erscheint so als eine des Kombinierens, des Zusammenfügens, des Verbindens schon vorhandener Bausteine. Der Schöpfungsakt ist folglich nicht notwendig ein Akt des Erfindens. Der Creacionismo impliziert eine Ästhetik, die keineswegs den vollständigen Bruch mit dem Vorherigen so radikal vollzieht, wie dies in manch einschlägiger Studie dargestellt wird. Es sind vielmehr die Anknüpfungen, die Fortentwicklungen, die Rückgriffe und das „invenire“, das (Vor-) Finden und weniger das (Er-) Finden, das den kreationistischen Dichter ausmacht.

Huidobros eigene Poetik basiert auf Dekomposition und Rekomposition, wenn auch nicht in jenem Sinne, den Huidobro ebenfalls in seinem Manifestes Manifeste angriff: Sie erinnern sich an die Gebrauchsanweisung Tristan Tzaras zur Verfertigung eines dadaistischen Gedichts? Es ging darum, die Schnipsel eines Zeitungsartikels auszuschneiden, zu mischen und sorgfältig in eine neue, zufällig durch das Schütteln erzeugte Ordnung zu bringen. Nichts aber hätte Huidobro ferner gelegen. Für ihn war der Kreator kein Schüttler, sondern im Grunde ein Demiurg in seinem Reich, in seinem weiten Universum.

So erscheint der Künstler und Dichter als ein kleiner Gott - eine Vorstellung, die wir freilich auch hier nicht als etwas völlig Neuartiges verstehen, sondern in ihren Bezügen zur romantischen Ästhetik beleuchten sollten. Denn im 19. Jahrhundert war der Sakralbegriff des ,Schöpfers‘ desakralisiert und für andere ,Schöpfungsprozesse‘ geöffnet worden, während umgekehrt die profane Figur des Dichters als Schöpfer neuer Welten sakralisiert wurde - ein „chassécroisé“, welches das 19. Jahrhundert in seiner Gesamtheit charakterisiert. Schütteln allein aber, dies war Huidobro klar, konnte den Dichter und die Dichtkunst nicht tragen und nicht weiterbringen.

Damit wandte sich Vicente Huidobro aber nicht nur gegen Tristan Tzara oder die französischen Surrealisten, sondern zugleich auch gegen eine Auflösung der Grenze zwischen Literatur und Lebensalltag, die den europäischen Avantgardisten so am Herzen lag. Genau an diesem Punkt scheint mir der grundlegende Unterschied zu den europäischen Avantgardebewegungen zu liegen. Denn Huidobro hielt fest am Konzept des großen Dichters, an Bild und Metaphysik des kleinen Gottes, der seine eigene, lyrische Welt zu schaffen in der Lage war. Er knüpfte damit nicht allein an Rubén Darío und dessen Rede von den „Torres de Dios“, sondern auch an José Enrique Rodó an, der in seinem nietzscheanisch 
geprägten El que vendrá auf die kommenden Philosophen oder Dichter anspielte, auf welche sich seine messianistischen Hoffnungen konzentrierten. Die Modernisten behielten dabei die Grenzen zwischen dem großen Poeten, dem großen Seher und der allgemeinen Masse des Volkes bei, wie dies etwa in der Schlussszene von Rodós Ariel zum Ausdruck kam. Vicente Huidobro veränderte an dieser Grundeinstellung nur wenige Elemente.

Kaum ein größerer Abstand ist hier denkbar zwischen der Position Huidobros und jener der Surrealisten. Dem Dichter kommt eine Vorreiterrolle, eine Avantgardeposition bezüglich der Versöhnung von Mensch und Welt im Sinne Huidobros zu. Auf der Ebene der Rolle des Dichters situiert sich auch die Verbindung zur „poésie pure“, die in allen kreationistischen Äußerungen und Konzeptionen Huidobros mitschwingt. Eine Öffnung oder gar Demokratisierung der Kunst ist von dieser Position nicht zu erwarten.

Auch das 1931 erstmals veröffentlichte Manifest Total legt, trotz derartiger Interpretationsversuche, keineswegs eine Wende in Richtung auf (gesamtgesellschaftlich fällige) Demokratisierungsbestrebungen nahe, wie sie im Übrigen sehr wohl innerhalb des Modernismo Martíscher Prägung zu beobachten waren. Im Gegenteil: Dieses zweifellos stärker politische Engagement ist durchaus für eine soziopolitische Totalisierung offen. Auch wenn dies über die Dichtkunst Huidobros wenig aussagen mag: von der Sakralisierung des Dichters ist es nur ein kleiner Schritt zu dessen totaler oder totalitärer Inthronisierung. Dies wirft zweifellos einen Schatten auf die Konzeptionen des Chilenen.

An dieser Stelle muss man abschließend aber unbedingt hinzufügen, dass das zuvor erörterte Vor-Finden, die Kombinatorik von Dekomposition und Rekomposition, die Bezugnahme und Transformation von bereits vorhandenen Textmaterialien, die - wie Huidobro selbst sagte - Verwandlung des Abstrakten in Konkretes (wie im Falle seines Gedichts Moulin) und des Konkreten in Abstraktes (wie im Falle des Manifestes Manifeste) sich nicht nur auf den hispanoamerikanischen, sondern ganz selbstverständlich auch auf den europäischen Raum bezieht. Daraus eine literarische oder kulturelle Dependenz, eine Abhängigkeit Lateinamerikas von Europa in Sachen Avantgarde, abzuleiten, scheint mir ebenso überzogen wie die schlichte Behauptung, all dies sei - wie es ebenfalls in einschlägigen Studien vorgetragen wird - ,típicamente latinoamericano'. Die lateinamerikanischen Literaturen sind in der ersten Hälfte des 20. Jahrhunderts sehr wohl auf dem Weg, die Asymmetrie der literarischen Beziehungen im Zeichen der Avantgarden zu ihren Gunsten zu verändern, auch wenn dies die Zeitgenossen in Europa bestenfalls allmählich zur Kenntnis nahmen. Auch auf dieser Ebene der internationalen und mehr noch transarealen Beziehungen stellten die lateinamerikanischen Avantgarden keinen Bruch dar: Sie brachen nicht mit den Literaturen Europas, sondern verleibten sich diese - wie etwa der brasilianische Modernismo, 
den man nicht mit dem hispanoamerikanischen verwechseln darf - bisweilen kannibalistisch ein. Bis heute ist das Konzept der Anthropophagie, das von der avantgardistischen Bewegung des brasilianischen Modernismo ins Spiel gebracht wurde, ebenso im Bereich der Kunst (wie eine der Anthropophagie gewidmete Biennale von São Paulo zeigt) wie im Feld der Literatur von größter Relevanz.

Mit pauschalen Behauptungen von einer mutmaßlichen Dependenz oder Annahmen, das sei eben ,typisch lateinamerikanisch', kommen wir nicht weiter. Vielmehr sehen wir, dass innerhalb unserer durchaus weit gefassten Anschauung und Definition von Avantgarde Vicente Huidobro zumindest in dieser Phase vor allem jener avantgardistischen Tendenz angehörte, die sich - wie in Mexiko Alfonso Reyes oder in Argentinien auch Oliverio Girondo - vor allem auf die Orientierung am Traditionsstrang der abendländischen Literaturen konzentrierte. Dabei nahm sie die Einbeziehung anderer kultureller Pole nicht vor oder verschob sie auf später. Zugleich aber eröffnete Vicente Huidobro - und hierin ist das Entscheidende seiner Kunst zu sehen - den lyrischen Ausdrucksformen der hispanoamerikanischen Literatur völlig neue Bereiche, schuf durch seine Experimente Zonen neuer Kreativität und führte in gewisser Weise die hispanoamerikanische Dichtkunst nicht nur auf der theoretischen, sondern vor allem auch auf der dichtungspraktischen Ebene an die Diskussionen der europäischen historischen Avantgarden heran. Er tat dies, ohne - wie man ihm fälschlich vorwarf - eine selbstreflexive Eigenposition vergessen und die spezifischen Bedingungen der Literatur in Lateinamerika übersehen zu haben. Mit Vicente Huidobro bestätigten die Literaturen Lateinamerikas, dass sie in einem weltweiten Zusammenhang auf der Höhe ihrer Zeit agierten. 